\title{
HIGHLIGHTS
}

METABOLISM

\section{Testosterone replacement therapy improves the metabolic syndrome}

Restoration or improvement of circulating testosterone concentrations in men with the metabolic syndrome improves variables related to this disorder, confirm the results of a placebo-controlled trial.

The metabolic syndrome is associated with a depression of testosterone levels, thereby raising the risk of cardiovascular disease and type 2 diabetes mellitus. "The approach taken in our research was to restore blood testosterone levels to the normal range and to monitor effects on the components of the metabolic syndrome," recounts author Louis J. Gooren (VU University Medical Center, Amsterdam).

Kalinchenko et al. enrolled 184 men aged $35-70$ years in a double-blind, placebo-controlled, phase III trial. Eligible participants had both the metabolic syndrome (defined by the International Diabetes Federation criteria) and hypogonadism (defined as a baseline total testosterone level $<12.0 \mathrm{nmol} / 1$ or a calculated free testosterone level
$<225 \mathrm{pmol} / \mathrm{l})$. The men were randomly allocated to receive either $1,000 \mathrm{mg}$ testosterone undecanoate $(n=113)$ or placebo $(n=71)$, administered at baseline and after 6 and 18 weeks.

\section{Restoring testosterone to normal in men with the metabolic syndrome is only one element... 77}

Modest, but significant, decreases in weight, BMI, waist circumference and levels of leptin and insulin were found after 30 weeks in the testosterone-treated group compared with the placebo group, whereas serum glucose levels and lipid profiles were unaltered.

"Research with testosterone versus placebo over extended periods is difficult, if not impossible, because men start to notice effects of testosterone thus impairing the blinding of the study," explains Gooren. A (no longer blinded) follow-up over an additional 36 weeks showed that the beneficial effects of testosterone normalization were progressive over at least 66 weeks. The study is still ongoing for a further 90 weeks.

Gooren suggests a holistic approach to the health problems of the aging male. "Restoring testosterone to normal in men with the metabolic syndrome is only one element in the approach to treatment. Changes in lifestyle (diet and exercise) are very helpful in treating the metabolic syndrome, and in fact will raise testosterone levels, but it is notoriously difficult to motivate elderly men to adopt a healthy lifestyle," he concludes.

Linda Koch

Original article Kalinchenko, S. Y. et al. Effects of testosterone supplementation on markers of the metabolic syndrome and inflammation in hypogonadal men with the metabolic syndrome: the double-blind placebo-controlled Moscow study. Clin. Endocrinol. (Oxf.) doi:10.1111/ j.1365-2265.2010.03845.x 